\title{
Effectiveness of Empirical Anti-pseudomonal Antibiotics Recurrent COPD Exacerbation: a Multicenter Retrospective Cohort Study
}

Akihiro Shiroshita ( $\square$ akihirokun8@gmail.com )

Ichinomiyanishi Hospital

Chisato Miyakoshi

Kobe City Medical Center General Hospital

Shunta Tsutsumi

Awa Regional Medical Center

Hiroshi Shiba

Kameda Medical Center

Chigusa Shirakawa

Kobe City Medical Center General Hospital

Kenya Sato

Saiseikai Yokohamashi Tobu Hospital

Shinya Matsushita

Saiseikai Yokohamashi Tobu Hospital

Yuya Kimura

National Hospital Organization Tokyo National Hospital

Keisuke Tomii

Kobe City Medical Center General Hospital

Masahiro Ohgiya

National Hospital Organization Tokyo National Hospital

Yuki Kataoka

Hyogo Prefectural Amagasaki General Medical Center

\section{Research Article}

Keywords: chronic obstructive pulmonary disease (COPD), Effectiveness, anti-pseudomonal antibiotics, exacerbation, retrospective cohort study

Posted Date: July 15th, 2021

DOl: https://doi.org/10.21203/rs.3.rs-714241/v1 
License: (c) (i) This work is licensed under a Creative Commons Attribution 4.0 International License. Read Full License 


\section{Abstract}

Although frequent chronic obstructive pulmonary disease (COPD) exacerbation has been associated with the isolation of Pseudomonas aeruginosa (PA) in sputum cultures, it remains unknown whether the empirical use of anti-pseudomonal antibiotics can improve outcomes in patients with frequent COPD exacerbations. This multicenter retrospective cohort study evaluated whether the empirical use of antipseudomonal antibiotics improves the hospital stay length in patients with recurrent COPD exacerbation (>2 admissions from April 1, 2008 to July 31, 2020). For statistical analysis, a log-linked Gamma model was used. Parameters were estimated using a generalized estimating equation model with an exchangeable correlation structure accounting for repeated observations from a single patient. Covariates included age, body mass index, home oxygen therapy use, respiratory rate, heart rate, oxygen use on admission, mental status, systemic steroid use, activities of daily living, and number of recurrences. Hospital-specific effects were specified as fixed effects. In total, 344 patients and 965 observations of recurrent COPD exacerbations were selected. Anti-pseudomonal antibiotics were used in 173 patients (18\%). The estimated change in hospital stay length between anti-pseudomonal and nonanti-pseudomonal antibiotics groups was 0.044 days [ $95 \%$ confidence interval; $-0.077,0.166]$. Thus, the use of empirical anti-pseudomonal antibiotics based only on recurrence may not be necessary.

\section{Introduction}

Chronic obstructive pulmonary disease (COPD) is one of the most common respiratory diseases [1]. Patients with COPD frequently experience acute exacerbations; the event rate has been reported as approximately 1-2 times per year [2]. Following COPD exacerbations, background factors such as body mass index, obstruction, dyspnea, and exercise capacity may worsen [3]. Moreover, previous hospitalization for COPD exacerbation has been reported to be an important prognostic factor in patients with COPD exacerbation $[4,5]$.

Antibiotics are used mainly in patients with moderate or severe exacerbations and/or cough and sputum purulence [6]. Initial empirical treatment with narrow-spectrum antibiotics, such as aminopenicillin with clavulanic acid, macrolide, and tetracycline, is recommended for the entire population with COPD exacerbation, based on previous systematic reviews [7, 8]. However, evidence that can guide decisions regarding whether to choose narrow-spectrum or broad-spectrum antibiotics is lacking. Based on expert opinions, local bacterial resistance patterns should be considered [6]. Furthermore, for patients with frequent exacerbations, anti-pseudomonal antibiotic use should be considered, as frequent COPD exacerbations have been reported as associated with the isolation of Pseudomonas aeruginosa (PA) [9]. However, it is unclear whether empirical treatment for PA can improve outcomes in patients with frequent COPD exacerbations [10]. Therefore, we aimed to assess the effectiveness of empirical anti-pseudomonal antibiotics in patients with recurrent COPD exacerbation. We hypothesized that anti-pseudomonal antibiotics would improve the length of the hospital stay in patients with frequent COPD exacerbation.

\section{Methods}




\section{Study design}

The study was designed as a multicenter retrospective cohort study across seven acute general hospitals in Japan: Awa Regional Medical Center, Hyogo Prefectural Amagasaki General Medical Center, Ichinomiyanishi Hospital, Kameda Medical Center, Kobe City Medical Center General Hospital, Saiseikai Yokohamashi Tobu Hospital, and Tokyo National Hospital. This study was conducted in accordance with the Declaration of Helsinki [11]. Additionally, this study was approved by the Institutional Review Board (IRB) of each hospital (registration number: 2020008), and the need for written informed consent was waived by the IRB of Ichinomiyanichi Hospital. This article was prepared in accordance with the Strengthening the Reporting of Observational Studies in Epidemiology (STROBE) Statement (Supplementary table 1) [12].

\section{Patient selection}

The patient enrolment period depended on the storage terms of the electronic medical records in each hospital during the study period of April 1, 2008 to July 31,2020. Inclusion criteria were age $\geq 40$ years and hospital admittance due to COPD exacerbation $\geq$ two times during the study period. Hospital admittance due to COPD exacerbation was determined by an admission-precipitating diagnosis of COPD exacerbation based on the 10th revision of the International Statistical Classification of Diseases and Related Health Problems (ICD-10) code (ICD-10 code: J44.1). The exclusion criteria were as follows: empirical use of anti-pseudomonal antibiotics for multidrug-resistant PA that are available in Japan (i.e. colistin, polymyxin B, and fosfomycin).

A validation study of the patient selection based on the ICD-10 code (J44.1) was conducted at Ichinomiyanishi Hospital, Kameda Medical Center, Saiseikai Yokohamashi Tobu Hospital, and Tokyo National Hospital during the study period. Patients who were selected according to the above inclusion criteria were reviewed using the clinical charts from a respiratory physician to confirm the diagnosis of COPD exacerbation.

\section{Data collection}

The following patient data were collected from the Diagnosis Procedure Combination database: age, admission date, discharge date, sex, height, weight, activities of daily living, comorbidities, tracheal intubation, and prognosis. Other patient data were collected through the review of electrical medical records: baseline COPD stage, type of inhaler used (inhaled corticosteroid, long-acting beta2-agonist, long-acting muscarinic antagonist), home oxygen therapy use, activities of daily living as assessed by the Barthel index, prior PA isolation, vital signs (systolic blood pressure, respiratory rate, heart rate), mental status, oxygen use on admission, systemic steroid therapy, and antibiotic therapy.

\section{Outcomes}

The primary outcome was the length of the hospital stay. The outcome was measured repeatedly for each patient, and observations were nested within individuals and hospitals. Thus, patients and hospitals 
were considered as clusters.

\section{Treatment of interest}

The treatment of interest comprised the empirical use of anti-pseudomonal antibiotics on admission or the next day, regardless of the dose and route of administration. Anti-pseudomonal antibiotics comprised drugs that are available in Japan, as follows: ceftazidime, cefozopran, cefepime, carbapenem (biapenem, doripenem, imipenem, and meropenem), piperacillin, piperacillin/tazobactam, aminoglycoside (gentamicin, tobramycin, and amikacin), quinolone (ciprofloxacin, levofloxacin, garenoxacin, and gatifloxacin), and aztreonam.

\section{Covariates}

Based on the previous literature, the following factors were selected as potential confounding factors: age, body mass index, home oxygen therapy use (binary data), respiratory rate, heart rate ( $\geq 109$ beats/min or not), oxygen use on admission (binary data), mental status (altered mental status or not), systemic steroid use on admission or the next day regardless of the dose (binary data), activities of daily living (Barthel index), and number of recurrences [4, 13-18].

\section{Statistical analysis}

Patient characteristics are summarized as numbers and percentages for categorical variables and as the median and interquartile range (IQR) for continuous variables. A log-linked Gamma model was used to evaluate the association between each variable and the length of the hospital stay [19]. Since repeated observations were obtained from a single patient, we used the generalized estimating equation method with an exchangeable correlation structure and robust standard error estimation. Hospital-specific effects were implemented as fixed effects.

Missing data were imputed using multiple imputations by chained equations on the assumption that data were missing at random [20]. Covariates of the outcome analysis (number of recurrences, age, home oxygen therapy use, oxygen use on admission, heart rate, respiratory rate, mental status, systemic steroid use, admitting hospital), the treatment variable (anti-pseudomonal antibiotic use), and the outcome variable (length of the hospital stay), in addition to systolic blood pressure as an auxiliary variable, were used to estimate the missing data [21, 22]. The results for each imputed dataset were aggregated using Rubin's rule [23].

We also constructed a Bayesian model in which both patient- and hospital-specific effects were implemented as random effects using the complete case dataset [24]. This model can be described as follows:

$Y_{i j k} \sim \operatorname{Gamma}\left(s, s / \mu_{i j k}\right)$

$\log \left(\mu_{i j k}\right)=X_{i j k} \beta+b_{j}+b_{k}$ 


$$
\sum b_{j}=0, \sum b_{k}=0
$$

where $Y_{i j k}$ is the length of the $i$-th stay of patient $j$ in hospital $k$, and the mean of the Gamma distribution, $\log (\mu)$, is determined by explanatory variables, $X$, and the patient- and hospital-specific effects with a zerosum constraint, $b_{j}$ and $b_{k}$. We set four separate sampling chains, each consisting of 5000 samples (including 4000 samples discarded for convergence). We evaluated the sampling convergence using the Gelman-Rubin statistic (R-hat) and visually inspected the trace plot.

All statistical analyses were performed using $R$ software version 4.0.2 (R Foundation for Statistical Computing, Vienna, Austria). For Bayesian model analyses, we used the probabilistic programming language, Stan (Stan Development Team). The script is available on GitHub (https://github.com/AkihiroShiroshita/Recurrent-COPD-AE) [25].

\section{Results}

The validation study conducted across four hospitals revealed that patient selection for recurrent COPD exacerbation using the ICD-10 code resulted in 6 of 108 false positives (positive predictive value of 94\%).

The patient selection flowchart is shown in Fig. 1. Among the selected 1574 patients with COPD exacerbation, 344 (22\%) were hospitalized with an admission-precipitating diagnosis of COPD exacerbation $\geq 2$ times during the study period (total number of observations/cases: 965 ). No patients were excluded because of the empirical use of anti-pseudomonal antibiotics for multidrug-resistant PA. The median number of recurrences was 3 (IQR: 2-4), and the median interval to the next hospitalization was 176 days (IQR: 55-507 days). The patients' characteristics are summarized according to treatment in Table 1. 
Table 1

Patient characteristics per observation

\section{Characteristic}

Non-antipseudomonal

antibiotics group

$\left(N^{*}=792,82 \%\right)$
Antipseudomonal

antibiotics group

$(N=173,18 \%)$

Demographic characteristics

Age (years, mean, $\mathrm{SD}^{\dagger}$ )

$78(9)$

$80(8)$

$78(9)$

Male (number, \%)

$697(88)$

$154(89)$

Height (cm, SD)

$160(11)$

$156(20)$

$53(12)$

$133(17)$

Missing data (number, \%)

$\mathrm{COPD}^{\ddagger}$ Stage

\(number, \%)

$36(9)$

2 (4)

$38(9)$

$\rrbracket$ (number, \%)

$120(31)$

$18(40)$

$138(32)$

$\nabla($ number, \%)

$155(40)$

15 (33)

$170(39)$

$\nabla($ number, \%)

Missing data (number, \%)

$79(20)$

$402(51)$

$288(36)$

$427(54)$

$493(62)$

$347(44)$

$45[10-80]$

$10(22)$

$89(21)$

Inhaled corticosteroid (number, \%)

Long-acting beta2-agonist (number, \%)

Long-acting muscarinic antagonist

(number, \%)

Home oxygen therapy users (number, \%)

Activities of daily living score (score, IQR§)

Missing data (number, \%)

$128(16)$

$3[3-4]$

IQR)

Median time to next hospitalization (days, 183 [58-533] IQR)

Total

$(\mathrm{N}=965)$

$160(10)$

$187(20)$

$52(12)$

$143(15)$

$530(55)$

366 (38)

$536(56)$

607 (63)

$114(66)$

413 (43)

66 (38)

45 [5-70]

30 [0-55]

$13(1)$

3 [2-4]

3 [3-5]

151 [53-424]

176 [55507] 


\begin{tabular}{|c|c|c|c|}
\hline Characteristic & $\begin{array}{l}\begin{array}{l}\text { Non-anti- } \\
\text { pseudomonal }\end{array} \\
\text { antibiotics group } \\
\left(\mathrm{N}^{*}=792,82 \%\right)\end{array}$ & $\begin{array}{l}\text { Anti- } \\
\text { pseudomonal } \\
\text { antibiotics } \\
\text { group } \\
(\mathrm{N}=173,18 \%)\end{array}$ & $\begin{array}{l}\text { Total } \\
(\mathrm{N}=965)\end{array}$ \\
\hline \multicolumn{4}{|l|}{ Comorbidities } \\
\hline Asthma (number, \%) & $149(19)$ & $39(23)$ & $188(20)$ \\
\hline Heart failure (number, \%) & $142(18)$ & $31(18)$ & $173(18)$ \\
\hline Cancer (number, \%) & $83(11)$ & $16(9)$ & $99(10)$ \\
\hline Diabetes mellitus (number, \%) & $107(14)$ & $35(20)$ & $142(15)$ \\
\hline \multicolumn{4}{|l|}{ Vital signs } \\
\hline Altered mental status (number, \%) & $105(13)$ & $29(17)$ & $134(14)$ \\
\hline $\begin{array}{l}\text { Systolic blood pressure (mmHg, mean, } \\
\text { SD) }\end{array}$ & $138(27)$ & $133(27)$ & $137(27)$ \\
\hline Missing data (number, \%) & $41(5)$ & $2(1)$ & $43(4)$ \\
\hline Heart rate (beats/min, mean, SD) & $99(20)$ & $105(22)$ & $100(20)$ \\
\hline Missing data & $30(4)$ & $4(2)$ & $34(4)$ \\
\hline Respiratory rate (breaths/min, mean, SD) & $24(6)$ & $26(7)$ & $25(6)$ \\
\hline Missing data (number, \%) & $62(8)$ & $18(10)$ & $80(8)$ \\
\hline Oxygen use on admission (number, \%) & $604(76)$ & $140(81)$ & $744(77)$ \\
\hline Missing data (number, \%) & $19(2)$ & $0(0)$ & $19(2)$ \\
\hline \multicolumn{4}{|l|}{ Treatment } \\
\hline Steroid therapy (number, \%) & $663(84)$ & $160(93)$ & $823(85)$ \\
\hline \multicolumn{4}{|l|}{ Prognosis } \\
\hline Length of hospital stay (days, IQR) & $11[8-17]$ & $12[9-21]$ & $12[8-18]$ \\
\hline Tracheal intubation (number, \%) & $39(5)$ & $17(10)$ & $56(6)$ \\
\hline Death (number, \%) & $37(5)$ & $18(10)$ & $55(6)$ \\
\hline
\end{tabular}

Abbreviations: *: $\mathrm{N}=$ number; $\mathrm{t}: \mathrm{SD}$ = standard deviation; $¥: \mathrm{COPD}$ = chronic obstructive pulmonary disease; $\S \mathrm{IQR}$ = interquartile range

9: Activities of daily living score is defined as the Barthel index. A high score indicates a higher activity level. 
PA was detected in at least 75 of 344 patients (22\%) during the study period. The strategies for empirical antibiotic use are summarized in Table 2 and Supplemental table 2. Piperacillin/tazobactam was used in $70 \%$ of anti-pseudomonal antibiotic cases, and ceftriaxone was used in $73 \%$ of non-anti-pseudomonal antibiotic cases. Although a sputum sample was not collected in all cases, at least 75 of 965 observations/cases ( $8 \%$ ) showed positive sputum-culture results for PA before admission (characterized in Supplemental table 3). Among these 75 cases, anti-pseudomonal antibiotics were used in 20 (27\%). None of the patients received double coverage with anti-pseudomonal antibiotics. Only $13 \%$ of cases received antibiotics covering atypical respiratory pathogens, including Mycoplasma pneumonia, Legionella spp., and Chlamydia spp. In the non-anti-pseudomonal antibiotics group, 18 of 474 cases (4\%) were switched to anti-pseudomonal antibiotics during hospitalization. 
Table 2

Empirical antibiotics therapy

\begin{tabular}{|c|c|}
\hline Antibiotics & Number \\
\hline \multicolumn{2}{|c|}{ Anti-pseudomonal antibiotics $(\mathrm{N}=173)$} \\
\hline Ceftazidime & 7 \\
\hline Cefozopran & 2 \\
\hline Cefepime & 16 \\
\hline Carbapenem & 6 \\
\hline Biapenem & 0 \\
\hline Doripenem & 0 \\
\hline Imipenem & 0 \\
\hline Meropenem & 6 \\
\hline Piperacillin & 4 \\
\hline Piperacillin/tazobactam & 120 \\
\hline Aminoglycoside & 1 \\
\hline Gentamicin & 0 \\
\hline Tobramycin & 0 \\
\hline Amikacin & 1 \\
\hline Quinolone & 13 \\
\hline Ciprofloxacin & 1 \\
\hline Levofloxacin & 11 \\
\hline Garenoxacin & 1 \\
\hline Gatifloxacin & 0 \\
\hline Aztreonam & 4 \\
\hline \multicolumn{2}{|c|}{ Non-anti-pseudomonal antibiotics $(\mathrm{N}=474)$} \\
\hline Amoxicillin & 5 \\
\hline Amoxicillin/clavulanic acid & 22 \\
\hline Ampicillin & 4 \\
\hline Cefuroxime & 1 \\
\hline Penicillin-G & 2 \\
\hline
\end{tabular}

Page 10/18 


\begin{tabular}{|ll|}
\hline Antibiotics & Number \\
\hline Sulbactam/ampicillin & 56 \\
\hline Cefotiam & 4 \\
\hline Cefotaxime & 2 \\
\hline Ceftriaxone & 345 \\
\hline Vancomycin & 11 \\
\hline Sulfamethoxazole/trimethoprim & 4 \\
\hline Macrolide & 64 \\
\hline Azithromycin & 46 \\
\hline Clarithromycin & 1 \\
\hline Erythromycin & 17 \\
\hline Tetracycline & 7 \\
\hline Minomycin & 6 \\
\hline Doxycycline & 1 \\
\hline Moxifloxacin & 1 \\
\hline
\end{tabular}

The main analysis with multiple imputations and the generalized estimating equation revealed that the estimated change in the length of the hospital stay between the anti-pseudomonal and non-antipseudomonal antibiotics groups was 0.044 days [ $95 \%$ confidence interval; $-0.077,0.166$ ] (Table 3$)$. This non-significant result was consistent with the results of the sensitivity analysis.

Table 3

The estimated difference in the length of the hospital stay between the anti-pseudomonal and non-antipseudomonal antibiotics groups

\section{Statistical model}

Coefficient

$95 \%$ confidence interval or $95 \%$ highest density interval

Multiple imputations and the generalized estimating equation with an exchangeable correlation structure

A complete case analysis and three-level Bayesian hierarchical model
$0.044 \quad-0.077,0.166$

0.178

$-1.836,2.061$

\section{Discussion}


This multicenter retrospective cohort study aimed to evaluate the effectiveness of empirical antipseudomonal antibiotics in terms of the length of the hospital stay in patients with frequent COPD exacerbation. We hypothesized that anti-pseudomonal antibiotics would reduce the length of the hospital stay. However, after adjusting for known confounding factors, the length of the hospital stay was not significantly different between the non-anti-pseudomonal and anti-pseudomonal antibiotics groups.

Although the PA detection rate in the present study was not lower than that in previous studies conducted in other countries ( $22 \%$ in the present study vs $4-13 \%)$, our hypothesis was not supported $[10,26]$. To the best of our knowledge, this study is the first to assess the effectiveness of anti-pseudomonal antibiotics in patients with recurrent COPD exacerbation. Our non-significant results are consistent with a previous prospective cohort study targeting hospitalized patients who had a positive culture result for PA in lowertract specimens [27]. In this previous study, only $18 \%$ of patients were treated with anti-pseudomonal antibiotics and, after adjusting for confounding factors, inadequate initial antibiotic use was not associated with 12-month mortality. In contrast, inappropriate initial antibiotic use in the context of PA pneumonia has been reported as associated with increased 28-day mortality [28]. This difference in results may be due to differences in the proportion of patients with pneumonia. In a study of PA pneumonia, the 28 -day mortality rate was $51 \%$, which is comparable to that in other reports [29]. Although PA detection among patients with COPD has been reported as associated with extremely high 2-year allcause mortality $(23-41 \%)$, the 1 -month mortality was $0 \%$ [10,30]. Considering the low 1 -month mortality in patients with COPD exacerbation with PA isolation, empirical anti-pseudomonal antibiotics may not have adequate power to change the prognosis during hospitalization.

Initial treatment with non-anti-pseudomonal antibiotics may spare anti-pseudomonal antibiotic use. The median time to the next hospitalization was approximately half a year, and the previous use of antibiotics was detected in only one-third of observations. In the present study, although at least 70 observations/cases had positive sputum-culture results for PA before admission, PA was covered by empirical antibiotics in only $29 \%$ of cases. The patient characteristics and length of the hospital stay were not substantially different between anti-pseudomonal and non-anti-pseudomonal antibiotics groups. Furthermore, a change in the treatment from non-anti-pseudomonal antibiotics to antipseudomonal antibiotics occurred in only $4 \%$ of cases. Thus, physicians may not need to administer antipseudomonal antibiotics based only on the number of recurrences. Currently, multidrug-resistant organisms are a global concern [31]. The judicious use of broad-spectrum antibiotics can reduce the number of drug-resistant microorganisms [32,33]. Additionally, the use of broad-spectrum antibiotics is associated with a high cost of care and increased antibiotic-associated side effects, such as Clostridium difficile infection [34]. Therefore, a strategy of empirical narrow-spectrum antibiotic use in patients with recurrent COPD exacerbation may be an acceptable choice.

This multicenter retrospective study has numerous strengths. First, this study was based on daily clinical practice. In contrast to the GOLD 2020 report and a network meta-analysis of randomised controlled trials (RCTs), the antibiotics used in the present cohort were mainly intravenous antibiotics [6,35]. This may be due to the large number of elderly patients requiring some assistance; the average age of the present 
cohort was 80 years, and only 175 of 965 (18\%) cases were fully independent. The present study results may better reflect hospitalized patients in daily practice rather than selected patients in RCTs [8]. Second, the number of observations was large compared to that in previous RCTs. The number of included patients/observations was much larger than that in a meta-analysis of four RCTs assessing the effect of currently used antibiotics on the length of the hospital stay ( 965 observations in the present study vs 393) [8]. Furthermore, an ongoing RCT targeting patients with at least one hospitalization within the prior 12 months and the detection of PA in a sputum culture has an expected total sample size of 150 (for the comparison of the anti-pseudomonal antibiotics group and the placebo group) [36], which is far smaller than that in the present study. Thus, the present results may fill gaps not covered by these RCTs.

The main caveat regarding this study is that we only evaluated the length of the hospital stay. Although the length of the hospital stay is associated with the survival of hospitalized patients with COPD, as an outcome, it is short-term and soft. Based on the results of previous systematic reviews, hard outcomes such as in-hospital death and 30-day mortality could not be evaluated in the present study because of its sample size $[7,8]$. Moreover, the time to the next exacerbation could not be evaluated because of unmeasured confounding factors, such as post-admission baseline treatments for COPD. In a previous retrospective cohort study with unmeasured confounding factors, antibiotic use was associated with improvements in the long-term mortality and time to the next exacerbation [37]. Further large-scale studies are warranted to evaluate hard short-term and long-term outcomes [38].

The present study had several other limitations. First, although our validation study showed a high predictive value for our patient selection strategy, the number of patients with COPD exacerbation was relatively small, considering that all of the hospitals were acute care and educational hospitals. Patient selection based on the ICD-10 code could have low sensitivity, and there could be many patients who should have been included in this study. In Japan, especially in our hospitals, payment is based on Diagnosis Procedure Combination: a system that reimburses hospitals based on the diagnosis code of hospitalized patients. Patients with a long length of stay or complications during hospitalization might be coded with diagnoses other than COPD exacerbation. Thus, the length of stay may have been righttruncated, and the effect of anti-pseudomonal antibiotics may have been skewed toward the null. Second, the interval between COPD exacerbations was not taken into account, and the variance correlation of the length of the hospital stay within each patient over multiple visits may differ from our expectations. We coped with this issue by using robust variances. Third, there may have been additional confounding factors. For example, because of the substantial amount of missing COPD stage data, we could not use it as a covariate; accordingly, the estimate of the effect of anti-pseudomonal antibiotics might have been skewed toward the null. Although we used other similar baseline covariates, such as the activities of daily living and home oxygen therapy use, an additional confounding factor may have altered the results. Further RCTs are needed to address unknown confounding factors.

\section{Conclusions}


The results of the present study suggest that empirical anti-pseudomonal antibiotics do not decrease the length of the hospital stay. Thus, empirical anti-pseudomonal antibiotic use based only on recurrence may not be necessary. However, further studies with larger sample sizes are needed to evaluate the effectiveness of anti-pseudomonal antibiotics more precisely.

\section{Declarations}

Acknowledgements. The authors would like to thank Hiroyuki Suzuki at the Department of Infectious Diseases of lowa University in the United States for providing us with a suggestion on multidrug-resistant bacteria.

Author contributions. AS, ST, HS, CS, KS, SM, Yuya K, KT, and Yuki Kataoka contributed to the conception and design of the work. AS, ST, HS, CS, KS, SM, Yuya K, KT, and MO contributed to data acquisition. AS, $\mathrm{CM}$, and Yuki Kataoka contributed to the data analysis and interpretation. AS, CM, and Yuki Kataoka drafted the manuscript. All authors revised the manuscript critically and approved the final version of the manuscript. AS, CM, ST, HS, CS, KS, SM, Yuya K, KT, MO, and Yuki K agreed to be accountable for all aspects of any part of the work.

Competing interests: The authors declare no competing interests.

Data availability statement. The datasets generated and/or analyzed during the current study are not publicly available due to the privacy issues but are available from the corresponding author on reasonable request.

Funding statement. Funding for English-language editing was obtained from the Systematic Review Workshop Peer Support Group (SRWS-PSG: https://community.camp-fire.jp/projects/view/187310). The funder played no role in the study design, study execution, data analyses, data interpretation, or decision to submit the results.

\section{References}

1. Halbert, R. J., Isonaka, S., George, D. \& Iqbal, A. Interpreting COPD prevalence estimates: what is the true burden of disease? Chest 123, 1684-1692 (2003). https://doi.org/10.1378/chest.123.5.1684

2. Hurst, J. R. et al. Susceptibility to exacerbation in chronic obstructive pulmonary disease. N. Engl. J. Med. 363, 1128-1138 (2010). https://doi.org/10.1056/NEJMoa0909883

3. Cote, C. G., Dordelly, L. J. \& Celli, B. R. Impact of COPD exacerbations on patient-centered outcomes. Chest 131, 696-704 (2007). https://doi.org/10.1378/chest.06-1610

4. Echevarria, C. et al. The PEARL score predicts 90 -day readmission or death after hospitalisation for acute exacerbation of COPD. Thorax 72, 686-693 (2017). https://doi.org/10.1136/thoraxjnl-2016209298 
5. Matkovic, Z. et al. Predictors of adverse outcome in patients hospitalised for exacerbation of chronic obstructive pulmonary disease. Respiration 84, 17-26 (2012). https://doi.org/10.1159/000335467

6. Global initiative for chronic obstructive lung disease - GOLD. 2020 Gold Reports. https://goldcopd.org/gold-reports_(2020).

7. Dobler, C. C. et al. Pharmacologic therapies in patients with exacerbation of chronic obstructive pulmonary disease: a systematic review with meta-analysis. Ann. Intern. Med. 172, 413-422 (2020). https://doi.org/10.7326/M19-3007

8. Vollenweider, D. J., Frei, A., Steurer-Stey, C. A., Garcia-Aymerich, J. \& Puhan, M. A. Antibiotics for exacerbations of chronic obstructive pulmonary disease. Cochrane Database Syst. Rev. 10, CD010257 (2018). https://doi.org/10.1002/14651858.CD010257.pub2

9. Garcia-Vidal, C. et al. Pseudomonas aeruginosa in patients hospitalised for COPD exacerbation: a prospective study. Eur. Respir. J. 34, 1072-1078 (2009). https://doi.org/10.1183/09031936.00003309

10. Eklöf, J. et al. Pseudomonas aeruginosa and risk of death and exacerbations in patients with chronic obstructive pulmonary disease: an observational cohort study of 22053 patients. Clin. Microbiol. Infect. 26, 227-234 (2020). https://doi.org/10.1016/j.cmi.2019.06.011

11. World Medical Association. WMA Declaration of Helsinki - Ethical Principles for Medical Research Involving Human Subjects. https://www.wma.net/policies-post/wma-declaration-of-helsinki-ethicalprinciples-for-medical-research-involving-human-subjects_(2018).

12. von Elm, E. et al. The Strengthening the Reporting of Observational Studies in Epidemiology (STROBE) statement: guidelines for reporting observational studies. Ann Intern. Med. 147, 573-577 (2007). https://doi.org/10.1016/j.ijsu.2014.07.013

13. Tabak, Y. P., Sun, X., Johannes, R. S., Gupta, V. \& Shorr, A. F. Mortality and need for mechanical ventilation in acute exacerbations of chronic obstructive pulmonary disease: development and validation of a simple risk score. Arch. Intern. Med. 169, 1595-1602 (2009). https://doi.org/10.1001/archinternmed.2009.270

14. Wilson, D. O., Rogers, R. M., Wright, E. C. \& Anthonisen, N. R. Body weight in chronic obstructive pulmonary disease. The National Institutes of Health Intermittent Positive-Pressure Breathing Trial. Am. Rev. Respir. Dis. 139, 1435-1438 (1989). https://doi.org/10.1164/ajrccm/139.6.1435

15. Schols, A. M., Slangen, J., Volovics, L. \& Wouters, E. F. Weight loss is a reversible factor in the prognosis of chronic obstructive pulmonary disease. Am. J. Respir. Crit. Care Med. 157, 1791-1797 (1998). https://doi.org/10.1164/ajrccm.157.6.9705017

16. Quintana, J. M. et al. Predictive score for mortality in patients with COPD exacerbations attending hospital emergency departments. BMC Med. 12, 66 (2014). https://doi.org/10.1186/1741-7015-1266

17. Flattet, Y., Garin, N., Serratrice, J., Perrier, A., Stirnemann, J. \& Carballo, S. Determining prognosis in acute exacerbation of COPD. Int. J. Chron. Obstruct. Pulmon. Dis. 12, 467-475 (2017). https://doi.org/10.2147/COPD.S122382 
18. Walters, J. A., Tan, D. J., White, C. J., Gibson, P. G., Wood-Baker, R. \& Walters, E. H. Systemic corticosteroids for acute exacerbations of chronic obstructive pulmonary disease. Cochrane Database Syst. Rev. 9, CD001288 (2014). https://doi.org/10.1002/14651858.CD001288.pub4

19. Williford, E., Haley, V., McNutt, L. A. \& Lazariu, V. Dealing with highly skewed hospital length of stay distributions: The use of Gamma mixture models to study delivery hospitalisations. PLoS One 15, e0231825 (2020). https://doi.org/10.1371/journal.pone.0231825

20. White, I. R., Royston, P. \& Wood, A. M. Multiple imputation using chained equations: Issues and guidance for practice. Stat. Med. 30, 377-299 (2011). https://doi.org/10.1002/sim.4067

21. He, Y. Missing data analysis using multiple imputation: getting to the heart of the matter. Circ. Cardiovasc. Qual. Outcomes 3, 98 (2010). https://doi.org/10.1161/CIRCOUTCOMES.109.875658

22. Moons, K. G., Donders, R. A., Stijnen, T. \& Harrell, F. E., Jr. Using the outcome for imputation of missing predictor values was preferred. J. Clin. Epidemiol. 59, 1092-1101 (2006). https://doi.org/10.1016/j.jclinepi.2006.01.009

23. Marshall, A., Altman, D. G., Holder, R. L. \& Royston, P. Combining estimates of interest in prognostic modelling studies after multiple imputation: current practice and guidelines. BMC Med. Res.

Methodol. 9, 57 (2009). https://doi.org/10.1186/1471-2288-9-57

24. Manandhar, B. \& Nandram, B. Hierarchical Bayesian models for continuous and positively skewed data from small areas. Commun. Stat. Theory Methods 50, 944-962 (2021). https://doi.org/10.1080/03610926.2019.1645853

25. R: The R Project for Statistical Computing. https://www.r-project.org_(2021).

26. Lieberman, D. \& Lieberman, D. Pseudomonal infections in patients with COPD: epidemiology and management. Am. J. Respir. Med. 2, 459-468 (2003). https://doi.org/10.1007/BF03256673

27. Eklöf, J., Gliese, K. M., Ingebrigtsen, T. S., Bodtger, U. \& Jensen, J. S. Antibiotic treatment adequacy and death among patients with Pseudomonas aeruginosa airway infection. PLoS One 14, e0226935 (2019). https://doi.org/10.1371/journal.pone.0226935

28. Park, S. Y. et al. Impact of adequate empirical combination therapy on mortality from bacteremic Pseudomonas aeruginosa pneumonia. BMC Infect. Dis. 12, 308 (2012). https://doi.org/10.1186/1471-2334-12-308

29. Fujitani, S., Sun, H. Y., Yu, V. L. \& Weingarten, J. A. Pneumonia due to Pseudomonas aeruginosa: Part I: epidemiology, clinical diagnosis, and source. Chest 139, 909-919 (2011). https://doi.org/10.1378/chest.10-0166

30. Rodrigo-Troyano, A. et al. Pseudomonas aeruginosa in chronic obstructive pulmonary disease patients with frequent hospitalised exacerbations: a prospective multicentre study. Respiration 96, 417-424 (2018). https://doi.org/10.1159/000490190

31. World Health Organization. Antimicrobial resistance 2020. https://www.who.int/healthtopics/antimicrobial-resistance (2020).

32. Lewis, G. J., Fang, X., Gooch, M. \& Cook, P. P. Decreased resistance of Pseudomonas aeruginosa with restriction of ciprofloxacin in a large teaching hospital's intensive care and intermediate care units. 
Infect. Control Hosp. Epidemiol. 33, 368-373 (2012). https://doi.org/10.1086/664763

33. Stultz, J. S., Arnold, S. R., Shelton, C. M., Bagga, B. \& Lee, K. R. Antimicrobial stewardship impact on Pseudomonas aeruginosa susceptibility to meropenem at a tertiary pediatric institution. $\mathrm{Am}$. J. Infect. Contro/ 47, 1513-1515 (2019). https://doi.org/10.1016/j.ajic.2019.05.001

34. Webb, B. J., Sorensen, J., Jephson, A., Mecham, I. \& Dean, N. C. Broad-spectrum antibiotic use and poor outcomes in community-onset pneumonia: a cohort study. Eur. Respir. J. 54, 1900057 (2019). https://doi.org/10.1183/13993003.00057-2019

35. Zhang, H. L., Tan, M., Qiu, A. M., Tao, Z. \& Wang, C. H. Antibiotics for treatment of acute exacerbation of chronic obstructive pulmonary disease: a network meta-analysis. BMC Pulm. Med. 17, 196 (2017). https://doi.org/10.1186/s12890-017-0541-0

36. Eklöf, J. Targeted AntiBiotics for Chronic Obstructive Pulmonary Disease (Target-ABC). https://clinicaltrials.gov/ct2/show/NCT03262142_(2020).

37. Roede, B. M., Bresser, P., Prins, J. M., Schellevis, F., Verheij, T. J. \& Bindels, P. J. Reduced risk of next exacerbation and mortality associated with antibiotic use in COPD. Eur. Respir. J. 33, 282-288 (2009). https://doi.org/10.1183/09031936.00088108

38. Gunen, H. et al. Factors affecting survival of hospitalised patients with COPD. Eur. Respir. J. 26, 234241 (2005). https://doi.org/10.1183/09031936.05.00024804

\section{Figures}

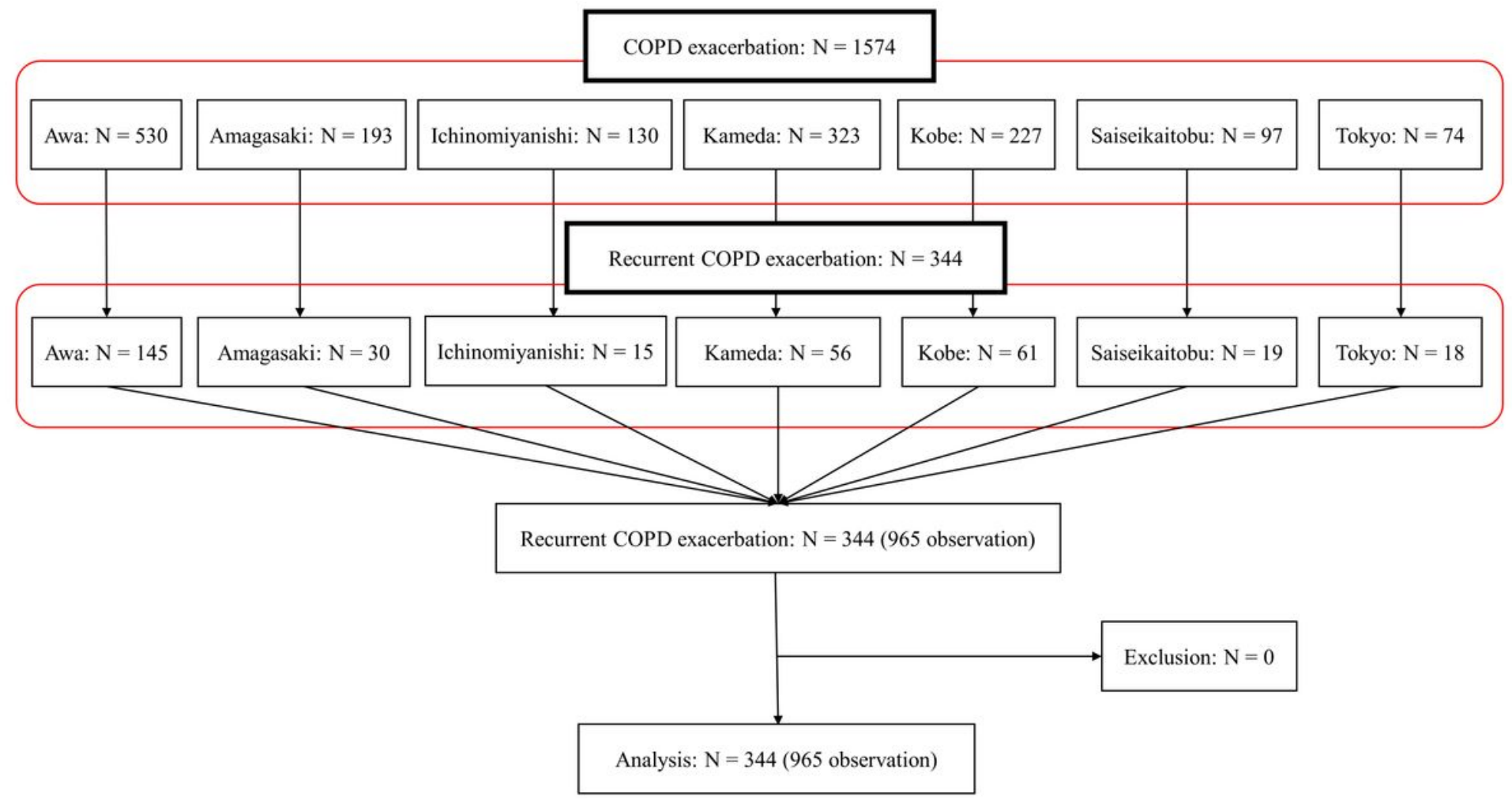

Figure 1 
Patient selection flowchart. COPD = chronic obstructive pulmonary disease

\section{Supplementary Files}

This is a list of supplementary files associated with this preprint. Click to download.

- Supplementaryfile.pdf 